\title{
Universal dimensions of simple Lie algebras and configurations of points and lines
}

\author{
Maneh Avetisyan ${ }^{a, *}$ \\ ${ }^{a}$ Yerevan Physics Institute, \\ Alikhanyan Brothers 2, Yerevan, Armenia \\ E-mail: maneh.avetisyan@gmail.com
}

The research, conducted by Vogel in 1999, in which the tensor category, called universal Lie algebra was introduced, provided a parametrization of the simple Lie algebras by three so-called universal parameters $(\alpha: \beta: \gamma)$ - projective coordinates in Vogel plane.

Subsequently, it has been shown, that several characteristics of simple Lie algebras, such as dimensions of certain representations, can be expressed in terms of these three parameters by some analytic functions, which are called universal formulae.

We investigate the uniqueness of the known universal dimension formulae, i.e. the possibility of the derivation of two different functions, yielding the same outputs at the same distinguished points. We employ the recently revealed geometrical rephrasing of this problem, which links us to a completely different area of mathematics - the theory of configurations of points and lines, particularly, we derive an explicit expression for a four-by-four non-uniqueness factor, making use of a known $\left(16_{3}, 12_{4}\right)$ configuration, demonstrating the benefit the geometrical interpretation provides with.

RDP online workshop "Recent Advances in Mathematical Physics" - Regio2020,

5-6 December 2020

online

\footnotetext{
${ }^{*}$ Speaker
} 


\section{Introduction}

Present research is rooted in the notion of universal Lie algebra, introduced by Vogel [1]. The latter is a certain tensor category having Vogel plane as a moduli space with special points corresponding to all simple Lie algebras. Recall that Vogel plane is the quotient space $P^{2} / S_{3}$ of projective plane with projective coordinates $(\alpha: \beta: \gamma)$ (Vogel's parameters) by the symmetric group $S_{3}$ acting by permutations of the parameters. The projective nature of the parameters corresponds to the choice of the invariant bilinear form on simple Lie algebra, which is known to be unique up to a multiple. The parameters corresponding to simple Lie algebras are given below, where the normalization corresponds to $\alpha=-2$.

Several characteristics of simple Lie algebras can be expressed in terms of only three Vogel's parameters by some formulae: these are called universal formulae.

An example of a universal formula is the following one, that is for the dimension of the $X_{2}$ representation:

$$
\operatorname{dim} X_{2}=\frac{(2 \alpha+\beta+\gamma)(\alpha+2 \beta+\gamma)(2 \alpha+2 \beta+\gamma)(\alpha+\beta+2 \gamma)(2 \alpha+\beta+2 \gamma)(\alpha+2(\beta+\gamma))}{\alpha^{2} \beta^{2} \gamma^{2}}
$$

which appears in the following universal decomposition of the antisymmetric square of the adjoint representation for each of the simple Lie algebra:

$$
\wedge^{2} \mathfrak{g}=\mathfrak{g} \oplus X_{2}
$$

A number of universal formulae have been derived both in the scope of the representation theory and in physical gauge theories, constructed on simple Lie algebras: [1-7]. Let's present an example [2]:

$$
\begin{gathered}
\operatorname{dim}\left((\mathfrak{g})^{2}\left(Y_{2}(\beta)\right)^{2}\right)=-\frac{(\alpha+\gamma)(2 \gamma-\alpha)(\beta+\gamma)(2 \beta+\gamma)(\beta+2 \gamma)(-\alpha+\beta+\gamma)(\alpha+\beta+\gamma)^{2}}{\alpha^{4} \beta^{2} \gamma} \times \\
\frac{(2 \alpha+\beta+\gamma)(-\alpha+2 \beta+\gamma)(\alpha+2 \beta+\gamma)(2 \alpha+2 \beta+\gamma)(\alpha-\beta+2 \gamma)(2 \alpha-\beta+2 \gamma)}{(\beta-4 \alpha)(\beta-3 \alpha)(\beta-\alpha)^{3}(2 \beta-3 \alpha)(\gamma-3 \alpha)} \times \\
\frac{(-3 \alpha+\beta+2 \gamma)(\alpha+\beta+2 \gamma)(2 \alpha+\beta+2 \gamma)(-5 \alpha+2 \beta+2 \gamma)(-\alpha+2 \beta+2 \gamma)(\alpha+2 \beta+2 \gamma)}{(\gamma-2 \alpha)(\gamma-\alpha)^{2}(2 \gamma-3 \alpha)(\gamma-\beta)(\alpha+\beta-\gamma)}
\end{gathered}
$$

where $Y_{2}(\beta)$ is one of the representation, appearing in the following universal decomposition of the symmetric square of the adjoint representation:

$$
S^{2} \mathfrak{g}=\mathbb{1} \oplus Y_{2}(\alpha) \oplus Y_{2}(\beta) \oplus Y_{2}(\gamma)
$$

As we see, the first formula writes much simpler than the latter. So, a natural question arises: can we find a more "simple-looking" formula, with the same features of universal nature? Or, generally, are the known universal formulae unique?

Establishing a geometrical rephrasing of this problem (see [8]), we find ourselves in a completely different area of mathematics - the theory of configurations of points and lines! In particular, we set up a fascinating correspondence between a three-by-three non-uniqueness factor [8] and the famous Pappus-Brianchon-Pascal configuration, which has been known since the 4-th century AD, as well as between a four-by-four non-uniqueness factor and one of the $\left(16_{3} 12_{4}\right)$ [9] configurations. 
Here we are going to demonstrate the correspondence procedure by carrying it out similarly as in [8], using another geometrical $\left(16_{3} 12_{4}\right)$ configuration; we derive a four-by-four non-uniqueness factor, using the necessary information, taken from the configuration itself.

We present the general expression for arbitrary non-uniqueness factor [8] along with the equations, satisfied by the parameters contained in it in Section 1.

Then in Section 2 we solve those equations to obtain a four-by-four non-uniqueness factor, using the data, provided by the $\left(16_{3} 12_{4}\right)$ configuration, presented in Figure 1 [9].

The outline of the ultimate situation of the problem of uniqueness, as well as its formulation in the scope of the theory of configurations is discussed in Conclusion.

\section{Non-uniqueness factors for universal quantum dimensions: general expression}

Let $F_{1}$ and $F_{2}$ be two universal formulae, which are rational functions, where both the numerator and denominator decompose into products of the same finite number of linear factors of Vogel's parameters, and yield the same outputs at the points from Table 1, which correspond to the simple Lie algebras.

Table 1: Vogel's parameters for simple Lie algebras and the distinguished lines

\begin{tabular}{|r|r|r|r|r|r|}
\hline Algebra/Parameters & $\alpha$ & $\beta$ & $\gamma$ & $t$ & Line \\
\hline$s l(N)$ & -2 & 2 & $N$ & $N$ & $\alpha+\beta=0$ \\
\hline$s o(N)$ & -2 & 4 & $N-4$ & $N-2$ & $2 \alpha+\beta=0$ \\
\hline $\operatorname{sp}(2 N)$ & -2 & 1 & $N+2$ & $N+1$ & $\alpha+2 \beta=0$ \\
\hline $\operatorname{exc}(n)$ & -2 & $2 n+4$ & $n+4$ & $3 n+6$ & $\gamma=2(\alpha+\beta)$ \\
\hline
\end{tabular}

On the exc line $n=-2 / 3,0,1,2,4,8$ for $G_{2}, s o(8), F_{4}, E_{6}, E_{7}, E_{8}$, respectively.

Then their ratio $Q$, which has the same structure, is obviously equal to 1 at those points.

$$
Q=\frac{F_{1}}{F_{2}}
$$

We call such functions $Q$ non-uniqueness factors. In fact, the problem of uniqueness of dimension formulae formulates as the search for possible non-uniqueness factors $Q$.

Note that the points from Vogel's table occupy the following distinguished lines [2]:

$$
\begin{array}{r}
s l: \alpha+\beta=0, \\
\text { so }: 2 \alpha+\beta=0, \\
s p: \alpha+2 \beta=0, \\
\text { exc }: \gamma-2(\alpha+\beta)=0,
\end{array}
$$

on which the linear, orthogonal, symplectic and the exceptional algebras are situated, respectively. Based on this fact, we add an additional requirement to the problem, namely, we search for a $Q$, which is equal to 1 not only at the points, associated with the simple Lie algebras, but also on the entire distinguished lines.

In [8] we have derived the following general expression for such non-uniqueness factors, equivalent to 1 on each of the four distinguished lines: 


$$
\begin{aligned}
& Q=\prod_{i=1}^{k} \frac{n_{i} \alpha+x_{i} \beta+y_{i} \gamma}{k_{i} n_{s(i)} \alpha+x_{i} \beta+y_{i} \gamma}=\prod_{i=1}^{k} \frac{n_{i} \alpha+x_{i} \beta+y_{i} \gamma}{c_{i} n_{p(i)} \alpha+x_{i} \beta+y_{i} \gamma} \\
& x_{i}=c_{i} x_{p(i)} \\
& y_{i}=k_{i} y_{s(i)} \\
& k_{i} n_{s(i)}=c_{i} n_{p(i)} \\
& y_{i}=r_{i} y_{v(i)} \\
& c_{i} n_{p(i)}+3 x_{i}=r_{i}\left(n_{v(i)}+3 x_{v(i)}\right) \\
& c_{1} c_{2} \ldots c_{k}=1 \\
& k_{1} k_{2} \ldots k_{k}=1 \\
& r_{1} r_{2} \ldots r_{k}=1
\end{aligned}
$$

for some permutations $s(i), p(i), v(i), i=1,2 \ldots k$. Note, that this expression is written after the following change of coordinates was made:

$$
\alpha^{\prime}=\alpha+\beta, \beta^{\prime}=2 \alpha+\beta \gamma^{\prime}=\gamma-2(\alpha+\beta)
$$

so that in the primed coordinates the equations of the distinguished lines $s l$, so, exc will simply be

$$
\alpha^{\prime}=0, \beta^{\prime}=0, \gamma^{\prime}=0
$$

respectively, and thus the one for the $s p$ line will be $3 \alpha^{\prime}-\beta^{\prime}=0$. The prime mark in (9) is dropped for convenience.

In order to write a non-uniqueness factor explicitly, one has to choose an appropriate set of the $s(i), p(i), v(i), i=1,2 \ldots k$ permutations, then solve the presented equations, using it. It is easy to see, that the choice of the set of permutations is quite wide: there are generally $k$ ! variations for each of them. Remarkably, there is a rather smart option of derivation of an explicit expression for some concrete $k$, which opens up after interpreting the non-uniqueness factors geometrically. In the next section we outline the geometrical rephrasing of the problem, then derive a four-by-four (at $k=4)$ non-uniqueness factor, invoking a specific $\left(16_{3} 12_{4}\right)$ geometrical configuration [9].

\section{Derivation of a non-uniqueness factor $Q$ using a configuration}

The essence of geometrical interpretation of the non-uniqueness factors, and generally, the universal dimension formulae, lies in the observation, that each of the linear factors of the universal formula determines a specific line in the projective plane [8]. So, for any non-uniqueness factor one can draw a defining sketch, consisting of $2 k$ lines, corresponding to each of its factors, and examine their relationship to the distinguished lines 5 . For convenience we refer to the lines, corresponding to the numerator of $Q$ as red lines, to those from the denominator - as green lines, and finally, to the distinguished ones - as black lines. Rephrasing the cancellation mechanism, necessary for $Q$ to be equivalent to 1 on each of the distinguished lines (see [8]), we observe the following specificity of distribution of the considered lines: each of the black lines must contain exactly $k$ points at which a green and a red line intersect. 


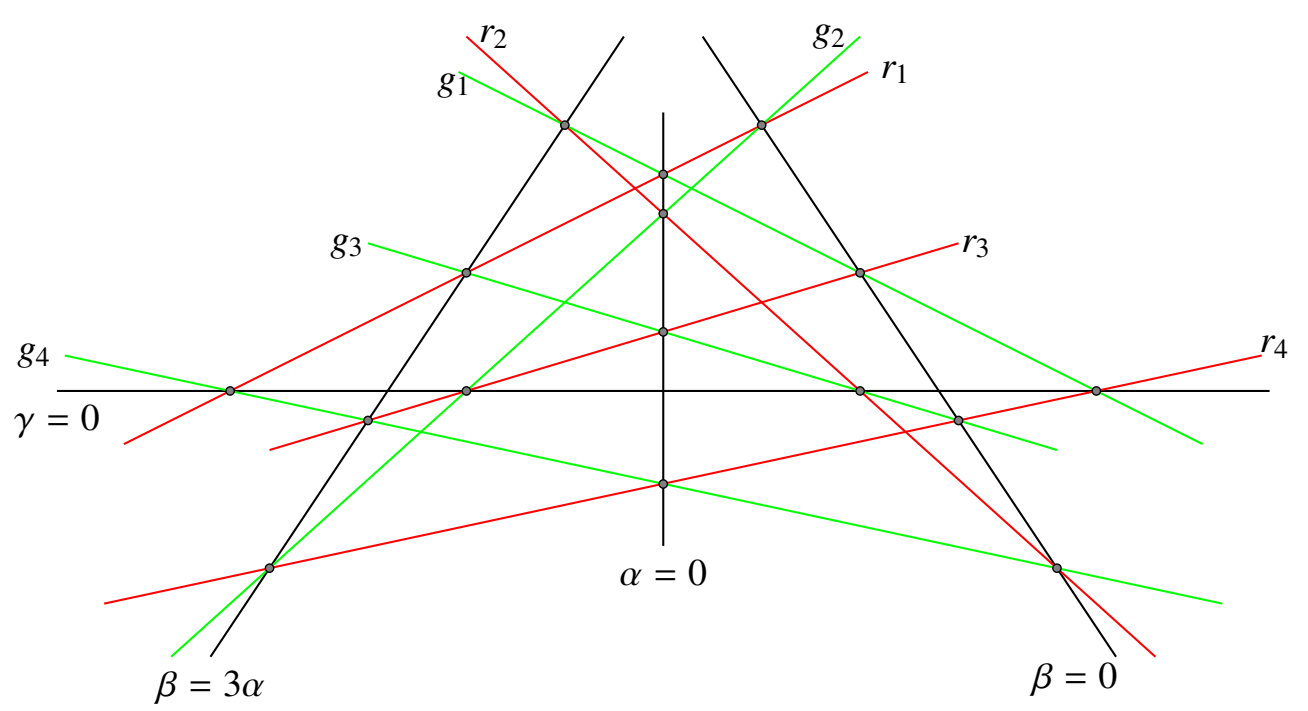

Figure 1: A $\left(16_{3} 12_{4}\right)$ configuration

Then we notice, that if the number of black lines is equal to the number of both red and green lines ${ }^{1}$, we will be dealing with a configuration of points and lines, more exactly, a $\left(k_{3}^{2}, 3 k_{k}\right)$ configuration: consisting of $k^{2}$ points at which exactly 3 lines of different colors intersect, and $3 k$ lines, on which exactly $k$ points lie (see $[9,10]$ ). Notice, that the existence of a configuration does not guarantee a corresponding non-uniqueness factor: it has to be possible to "color" the lines, such that at each of the points of the configuration lines of different colors meet. ${ }^{2}$

In [8] we present a three-by-three $(k=3$, i.e. we take three black lines, namely $s l$, so, exc) non-uniqueness factor, corresponding to the famous Pappus-Brianchon-Pascal $\left(9_{3}\right)$ configuration [10], as well as derive a four-by-four $(k=4)$ non-uniqueness factor, taking the necessary information, namely the set of the $s(i), p(i), v(i), i=1,2 \ldots k$ permutations, from a known geometrical configuration $\left(16_{3}, 12_{4}\right)$.

Here we take a different $\left(16_{3}, 12_{4}\right)$ configuration, and derive another non-uniqueness factor at $k=4$.

Let us consider the configuration, presented in Figure 1.

As it is shown, it can be colored in a needed way, so that it corresponds to some non-uniqueness factor $Q$ at $k=4$. Tracing the pairs of green and red lines, intersecting at each of the black lines, we almost immediately define the set of three permutations $s(i), p(i), v(i), i=1,2 \ldots k$ :

$$
\begin{aligned}
& s(1)=3, s(2)=1, s(3)=4, s(4)=2 \\
& p(1)=4, p(2)=3, p(3)=2, p(4)=1 \\
& v(1)=2, v(2)=4, v(3)=1, v(4)=3
\end{aligned}
$$

${ }^{1}$ Evidently, the number of red lines is equal to the number of green lines.

${ }^{2}$ Some examples of configurations, which cannot be colored in this manner, are presented in [8]. 
Then, equations (10)-(13) and (15)-(17) yield:

$$
\begin{array}{r}
x_{3}=\frac{x_{2}}{c_{2}}, x_{4}=\frac{x_{1}}{c_{1}} \\
y_{2}=k_{2} y_{1}, y_{3}=\frac{y_{1}}{k_{1}}, y_{4}=k_{2} k_{4} y_{1} \\
n_{2}=\frac{n_{1}}{c_{1} k_{4}}, n_{3}=\frac{k_{2} n_{1}}{c_{2}}, n_{4}=\frac{k_{1} k_{2} n_{1}}{c_{1} c_{2}} \\
r_{1}=1 / k_{2}, r_{2}=1 / k_{4}, r_{3}=1 / k_{1}, r_{4}=1 / k_{3} \\
c_{4}=\frac{1}{c_{1}}, c_{3}=\frac{1}{c_{2}}
\end{array}
$$

The remaining equation (14) yields:

$$
x_{1}=-\frac{k_{1} k_{2}+c_{2}}{3 c_{2}} n_{1}, x_{2}=-\frac{n_{1}}{c_{1} k_{4}}-k_{2} n_{1}
$$

So that we get the following non-trivial solution for $Q$ :

$$
\begin{array}{r}
Q=\frac{\left(3 k k_{1} k_{2} n \alpha+\left(-k k_{1} k_{2} n-k_{1} n\right) \beta+3 c_{2} k y \gamma\right)\left(3 n \alpha+n\left(-k k_{2}-1\right) \beta+3 k k_{2} y \gamma\right)}{\left(-3 k_{1} n \alpha+\left(k k_{1} k_{2} n+k_{1} n\right) \beta-3 c_{2} k y \gamma\right)\left(-3 c_{2} n \alpha+\left(c_{2} n+k_{1} k_{2} n\right) \beta-3 c_{2} k k_{2} y \gamma\right)} \times \\
\frac{\left(3 c_{2} n \alpha+\left(-c_{2} n-k_{1} k_{2} n\right) \beta+3 c_{2} y \gamma\right)\left(-3 k_{1} k_{2} n \alpha+\left(c_{2} n+k_{1} k_{2} n\right) \beta-3 c_{2} k k_{2} y \gamma\right)}{\left(3 k_{1} k_{2} n \alpha+\left(-c_{2} n-k_{1} k_{2} n\right) \beta+3 c_{2} y \gamma\right)\left(3 k k_{2} n \alpha+\left(-k k_{2} n-n\right) \beta+3 k k_{2} y \gamma\right)}
\end{array}
$$

where we put

$$
y=y_{1}, n=n_{1}, k=c_{1} k_{4} .
$$

Note, that the number of free parameters in (24) is 6 , whereas the same number in the nonuniqueness factor, derived in [8] is 4; this means that the (24) is richer in the sense, that it provides a wider range for choice of the parameters ${ }^{3}$.

Hence, we have derived an explicit expression (24) at $k=4$ for a non-uniqueness factor, invoking the geometrical interpretation of it and making use of the appropriate configuration $\left(16_{3}, 12_{4}\right)$ (Figure 1), thus demonstrating the practical role of geometrical interpretation of the uniqueness problem.

\section{Conclusion}

Ultimately, we presented the core achievements of the recent work [8], namely, the ideas, which led us to establish a correspondence between the problem of uniqueness of universal dimension formulae of simple Lie algebras and the theory of configurations. We demonstrated the practical advantages of the use of the configurations of points and lines by carrying out a derivation of an explicit expression for a different four-by-four non-uniqueness factor (24) making use of the information, provided by it.

Overall, this investigation, besides being important in the theory of simple Lie algebras, is remarkable in the sense, that it exposes a previously unnoticed relationship between two distinct concepts in mathematics - simple Lie algebras and configurations of points and lines.

\footnotetext{
${ }^{3}$ The geometrical interpretation of the free parameters is discussed $\mathrm{n}$ [8].
} 


\section{Acknowledgements}

This work was fulfilled within the Regional Doctoral Program on Theoretical and Experimental Particle Physics, sponsored by VolkswagenStiftung. It was also partially supported by the Science Committee of the Ministry of Science and Education of the Republic of Armenia under contracts 20AA-1C008 and 21T-1C105.

I am grateful to Professor R.Mkrtchyan for his encouraging discussions. I am also thankful to the organizers of RDP Online Workshop on Mathematical Physics (December 5-6, 2020) for invitation as well as to the participants of the workshop for showing interest in this work.

\section{References}

[1] P. Vogel, The Universal Lie algebra, Preprint (1999), https://webusers.imjprg.fr/ pierre.vogel/grenoble-99b.pdf

[2] J.M. Landsberg and L.Manivel, A universal dimension formula for complex simple Lie algebras, Adv. Math. 201 (2006), 379-407

[3] M.Y. Avetisyan and R.L. Mkrtchyan, $X_{2}$ Series of Universal Quantum Dimensions, arXiv:1812.07914, J. Phys. A: Math. Theor. Volume 53, Number 4, 045202, https://doi.org/10.1088/1751-8121/ab5f4d

[4] M.Y. Avetisyan and R.L. Mkrtchyan, On $(a d)^{n}\left(X_{2}\right)^{k}$ series of universal quantum dimensions, arXiv:1909.02076, J. Math. Phys. 61, 101701 (2020), https://doi.org/10.1063/5.0007028

[5] R.L. Mkrtchyan and A.P.Veselov, Universality in Chern-Simons theory, JHEP08, (2012), 153, arxiv:1203.0766

[6] R.L.Mkrtchyan, Nonperturbative universal Chern-Simons theory, JHEP09, (2013), 54, arxiv:1302.1507

[7] D. Krefl, R.Mkrtchyan, Exact Chern-Simons / Topological String duality, JHEP10, (2015), 45, arxiv: 1506.03907

[8] M.Y. Avetisyan and R.L. Mkrtchyan, Uniqueness of universal dimensions and configurations of points and lines, arxiv:2101.10860

[9] Grünbaum, Branko, Configurations of Points and Lines, Graduate Studies in Mathematics, 103, American Mathematical Society, ISBN 978-0-8218-4308-6, (2009).

[10] D.Hilbert, S.Cohn-Vossen Geometry and the Imagination, (2nd ed.), Chelsea, ISBN 0-82841087-9, (1952). 